\title{
Statistical properties of local active galactic nuclei inferred from the RXTE 3-20 keV all-sky survey ${ }^{\star}$
}

\author{
S. Yu. Sazonov ${ }^{1,2}$ and M. G. Revnivtsev ${ }^{1,2}$ \\ 1 Max-Planck-Institut für Astrophysik, Karl-Schwarzschild-Str. 1, 85740 Garching bei München, Germany \\ e-mail: sazonov@mpa-garching.mpg.de \\ 2 Space Research Institute, Russian Academy of Sciences, Profsoyuznaya 84/32, 117997 Moscow, Russia
}

Received 27 January 2004 / Accepted 16 April 2004

\begin{abstract}
We compiled a sample of 95 AGNs serendipitously detected in the 3-20 keV band at Galactic latitude $|b|>10^{\circ}$ during the RXTE slew survey (XSS, Revnivtsev et al. 2004), and utilize it to study the statistical properties of the local population of AGNs, including the X-ray luminosity function and absorption distribution. We find that among low X-ray luminosity $\left(L_{3-20}<\right.$ $10^{43.5} \mathrm{erg} \mathrm{s}^{-1}$ ) AGNs, the ratio of absorbed (characterized by intrinsic absorption in the range $10^{22} \mathrm{~cm}^{-2}<N_{\mathrm{H}}<10^{24} \mathrm{~cm}^{-2}$ ) and unabsorbed $\left(N_{\mathrm{H}}<10^{22} \mathrm{~cm}^{-2}\right)$ objects is 2:1, while this ratio drops to less than 1:5 for higher luminosity AGNs. The summed $\mathrm{X}$-ray output of AGNs with $L_{3-20}>10^{41} \mathrm{erg} \mathrm{s}^{-1}$ estimated here is smaller than the earlier estimated total X-ray volume emissivity in the local Universe, suggesting that a comparable X-ray flux may be produced together by lower luminosity AGNs, non-active galaxies and clusters of galaxies. Finally, we present a sample of 35 AGN candidates, composed of unidentified XSS sources.
\end{abstract}

Key words. galaxies: Seyfert - X-rays: general - galaxies: quasars: general

\section{Introduction}

We have recently (Revnivtsev et al. 2004, hereafter Paper I) taken advantage of the excellent calibration, moderate field of view (1 deg radius) and high effective area ( 6000 sq. $\mathrm{cm}$ ) of the PCA spectrometer on board the RXTE observatory to perform an all-sky survey in the 3-20 keV band from the data accumulated during satellite slews in 1996-2002 - the RXTE slew survey (XSS). For $90 \%$ of the sky at $|b|>10^{\circ}$, a flux limit for source detection of $2.5 \times 10^{-11} \mathrm{erg} \mathrm{s}^{-1} \mathrm{~cm}^{-2}(3-20 \mathrm{keV})$ or lower was achieved, while a combined area of $7 \times 10^{3}$ sq. deg was sampled to record flux levels (for such very large area surveys) below $10^{-11} \mathrm{erg} \mathrm{s}^{-1} \mathrm{~cm}^{-2}$.

In Paper I, a catalog comprising 294 X-ray sources detected at $|b|>10^{\circ}$ was presented. 236 of these sources were identified with a single known astronomical object. Of particular interest are 100 identified active galactic nuclei (AGNs) and 35 unidentified sources. The hard spectra of the latter suggest that many of them will probably also prove to be AGNs when follow-up observations are performed. Most of the detected AGNs belong to the local population $(z<0.1)$. In addition, the hard X-ray band of the XSS (3-20 keV) as compared to most previous $\mathrm{X}$-ray surveys, performed at photon energies below $10 \mathrm{keV}$, has made possible the detection of a substantial number of X-ray

* Tables 1 and 2 are only available in electronic form at the CDS via anonymous ftp to cdsarc.u-strasbg.fr $(130.79 .128 .5)$ or via http://cdsweb.u-strasbg.fr/cgi-bin/qcat?J/A+A/423/469 absorbed AGNs (mostly Seyfert 2 galaxies). These properties make the XSS sample of AGNs a valuable one for the study of the local population of AGNs.

In this paper, we carry out a thorough statistical analysis of the above sample to investigate several key properties of the local population of AGNs, in particular their distribution in intrinsic absorption column density $\left(N_{\mathrm{H}}\right)$ and X-ray luminosity function. Knowledge of these characteristics provides important constraints for AGN unification models and synthesis of the cosmic X-ray background, and is further needed to understand the details of the accretion-driven growth of supermassive black holes in the nuclei of galaxies.

In the course of the paper, we compare our results with previously published ones. These include the X-ray luminosity function of local AGNs derived from the HEAO-1/A2 allsky survey (Piccinotti et al. 1982), the $N_{\mathrm{H}}$ distribution of optically selected Seyfert 2 galaxies (Risaliti et al. 1999) and the evolving with redshift properties of AGNs inferred largely from medium-sensitivity and deep X-ray surveys (La Franca et al. 2002; Ueda et al. 2003; Steffen et al. 2003). Finally, we assess the contribution of AGNs with luminosities above $\sim 10^{41} \mathrm{erg} \mathrm{s}^{-1}$ to the total X-ray volume emissivity in the local Universe, as estimated by Miyaji et al. (1994).

\section{The sample}

There are 100 identified AGNs in the XSS catalog, of which 95 make up the input sample (Table 1) for the current study. 
One source (the radio galaxy $4 \mathrm{C}+21.55$ ) was excluded because its redshift is unknown. Another 4 objects (Mrk 335, Mrk 348, Ton S180 and NGC 1068) were excluded because they would not have satisfied the $4 \sigma$ detection criterion (in the 3-20 keV energy band) had there been no RXTE slews associated with pointed observations of these sources, i.e. they were not detectable serendipitously during the survey. Note that NGC 1068 is a Compton thick Seyfert 2 galaxy (i.e. having an X-ray spectrum characterized by an intrinsic absorption column density $N_{\mathrm{H}}>1.5 \times 10^{24} \mathrm{~cm}^{-2}$ ), and after its removal from the list we are left with just one source of this type - NGC 4945. Given this fact, we restrict the present analysis to AGNs with $N_{\mathrm{H}}<10^{24} \mathrm{~cm}^{-2}$, i.e. to Compton thin sources.

For each object in the sample a detailed AGN class is adopted from the XSS catalog, which in turn mostly follows the classification of the NED database. The sample includes 18 blazars. Their emission, including the X-rays, is collimated in our direction, which makes this class distinctly different from normal, emission-line AGNs. We have decided to consider the poorly studied source TEX $0222+185$ a blazar due to its extraordinary inferred X-ray luminosity $\left(\sim 10^{48} \mathrm{erg} \mathrm{s}^{-1}\right)$. The remaining 77 sources are non-blazar AGNs of various types, mostly Seyfert galaxies. Note that no strict division is drawn here between Seyfert galaxies and quasars; typically, AGNs designated as Seyferts (or radio galaxies) and quasars have an $\mathrm{X}$-ray luminosity below and above $10^{44.5} \mathrm{erg} \mathrm{s}^{-1}$, respectively.

In the non-blazar subsample, 60 objects are optically classified as type 1 AGNs and 7 of these are narrow-line Seyfert 1 galaxies (NLS1). We can directly infer from these numbers that in the local Universe NLS1 galaxies make up $\sim 10 \%$ of hard X-ray (3-20 keV) selected type 1 AGNs. This result fits well in the picture summarized by Grupe (2000) that NLS1 galaxies appear significantly enhanced (reaching $\sim 40 \%$ ) in soft $\mathrm{X}$-ray selected samples compared with hard X-ray selected ones. Radio loud AGNs (radio galaxies and radio-loud quasars) amount to $10 / 60 \sim 15 \%$ of type 1 objects in our sample, which is consistent with the well-known fraction $(\sim 10 \%)$ of radio loud objects among optically selected quasars (e.g. Ivezić et al. 2002). We note that it would be wrong to estimate here in the same straightforward way the proportion of unabsorbed and absorbed AGNs, which is one of the primary goals of this study, since our sample is biased against the latter type due to the sampled space volume decreasing with increasing $N_{\mathrm{H}}$ for a given intrinsic luminosity. The corresponding accurate calculation will be done in Sect. 3.

The information given in Table 1 for each AGN includes the measured count rates in two energy bands $3-8 \mathrm{keV}$ and 8-20 keV together with their $1 \sigma$ statistical uncertainties. We point out that these count rates have been obtained by averaging over multiple slews performed at random times during the period 1996-2002. It is important to note that in contrast to the original XSS catalog, the uncertainties quoted here do not take into account RXTE slews related to pointings at the sources. The current sample is thus effectively serendipitous. As was noted above, the corresponding correction has led to the removal of 4 AGNs from the sample. All of the presented AGNs are detected at a more than $4 \sigma$ confidence level in the 3-20 keV band. It should be noted that at the

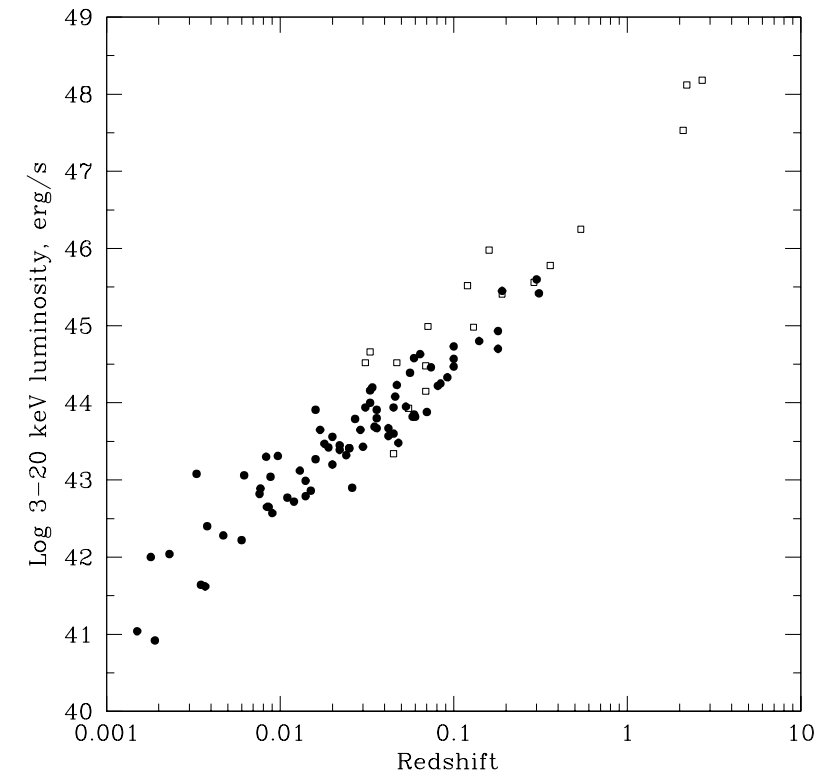

Fig. 1. Distribution in redshift and luminosity of 95 known AGNs detected in the RXTE slew survey, including 77 emission-line AGNs (solid circles) and 18 blazars (open squares).

faintest fluxes, source confusion may affect the count rate estimation. In Paper I a relevant threshold was estimated as $4 \sigma_{\text {conf }}=0.5 \mathrm{cnt} \mathrm{s}^{-1}$ for the $3-20 \mathrm{keV}$ band. Since only 3 of the AGNs in our sample have measured count rates below this limit, the overall effect of confusion on the sample is definitely negligible.

Next, two types of luminosity in the observer's $3-20 \mathrm{keV}$ band $^{1}$ are given for each source. The observed luminosity, $L_{3-20}$, is calculated from the measured 3-20 keV count rate (the sum of the $3-8 \mathrm{keV}$ and $8-20 \mathrm{keV}$ count rates) by taking into account the spectral response of the RXTE/PCA instrument and assuming a power-law spectrum of photon index $\Gamma=1.8$ with a low-energy cutoff due to intrinsic absorption (see Sect. 2.1 below). The intrinsic luminosity $L_{3-20 \text {,int }}$ is then found by correcting the observed luminosity for the intrinsic absorption. We also note that the quoted luminosities for the only AGN in our sample with $N_{\mathrm{H}}>10^{24} \mathrm{~cm}^{-2}-$ NGC 4945 - should be regarded as crude estimates because the X-ray spectrum of this Compton thick source is poorly described (Guainazzi et al. 2000) by the photoabsorbed power law model adopted here.

The luminosity distances were computed from the known redshifts assuming a cosmology with $\left(H_{0}, \Omega_{\mathrm{m}}, \Omega_{\Lambda}\right)=$ $\left(75 \mathrm{~km} \mathrm{~s}^{-1} \mathrm{Mpc}^{-1}, 0.3,0.7\right)$. For 10 nearby sources $(z \lesssim 0.01)$, the distances from the Nearby Galaxies Catalogue (Tully 1988) were adopted.

We show in Fig. 1 the location of our AGNs on the redshiftluminosity plane. One can see that the distribution in observed luminosity is extremely broad, spanning 7 (5) orders of magnitude if the blazars are included or not. We are effectively probing the local Universe $(z<0.1)$. These properties combined

\footnotetext{
${ }^{1}$ Defining the luminosities in the rest-frame 3-20 keV band makes essentially no difference, since the estimated k-correction $|\Delta \log L|<$ 0.1 for all the sources in the sample.
} 
with the hard X-ray range (3-20 keV) distinguish our survey from others.

\subsection{Absorption column density}

Another important property of AGNs is intrinsic absorption column density $\left(N_{\mathrm{H}}\right)$. It plays crucial roles in the AGN unification paradigm and in the study of the cosmic X-ray background. It should be noted that throughout this paper we ignore the contribution of Compton scattering to intrinsic extinction and consider photoabsorption only. This is justified because we do not consider Compton thick sources and since the effect of Compton scattering on the spectrum is expected to be small (less than $25 \%$ in terms of the observed $3-20 \mathrm{keV}$ flux) for $N_{\mathrm{H}}<10^{24} \mathrm{~cm}^{-2}$ (Yaqoob 1997; Matt et al. 1999) and further tends to be counteracted by the presence of a reflected spectral component (Matt et al. 2000; Risaliti 2002) neglected here.

For all of our identified or candidate (see Sect. 2.2) AGNs, the absorption column can be estimated to a first approximation from the ratio of the measured count rates in the $8-20 \mathrm{keV}$ and $3-8 \mathrm{keV}$ bands, assuming a $\Gamma=1.8$ power-law intrinsic spectrum and taking into account the source redshift (if known). The above spectral slope is typical for Seyfert galaxies and quasars, as is known from previous studies (e.g. Reynolds 1997) and also follows from our own analysis of pointed RXTE observations. Since NLS1 galaxies typically have somewhat softer $(\Gamma \approx 2.2)$ and unabsorbed spectra (e.g. Leighly 1999), the above procedure is expected to give the correct result $\left(N_{\mathrm{H}}=0\right)$ also for objects of this type. In this work, we do not distinguish column densities below $10^{22} \mathrm{~cm}^{-2}$, and therefore possible source-to-source variations of the order of 0.2 in the intrinsic power-law index should have no effect on our results. Interstellar absorption is similarly unimportant, since the XSS sources are located at $|b|>10^{\circ}$.

For most of the identified AGNs, we have been able to improve the above crude estimate of the absorption column either by analysing the spectral data of relevant pointed RXTE observations, or adopting $N_{\mathrm{H}}$ values from the TARTARUS/ASCA database or the literature. Data of RXTE/PCA pointed observations were reduced using the FTOOLS/LHEASOFT 5.3 package. The spectral modelling was done with XSPEC. AGN spectra were fitted by a simplistic model consisting of a power-law component photoabsorbed by a column of neutral material and a fluorescent neutral iron emission line at energy $6.4 \mathrm{keV}$, if the latter was required by the fit (model zphabs * (power + gaus) in XSPEC). For almost all the absorbed AGNs, the spectral fitting yielded values of the photon index $\Gamma \approx 1.8$, except in the well-known case of NGC $4151(\Gamma \approx 1.4)$ (see Schurch \& Warwick 2002, and references therein). The obtained $N_{\mathrm{H}}$ values were then compared with those estimated from the XSS catalog. The result of this comparison (Fig. 2) demonstrates that the photoabsorption column density can be robustly estimated from the XSS hardness ratio. We note that systematic uncertainties of the RXTE/PCA energy response do not allow one to measure absorption columns with an accuracy better than $(0.5-1) \times$ $10^{22} \mathrm{~cm}^{-2}$.

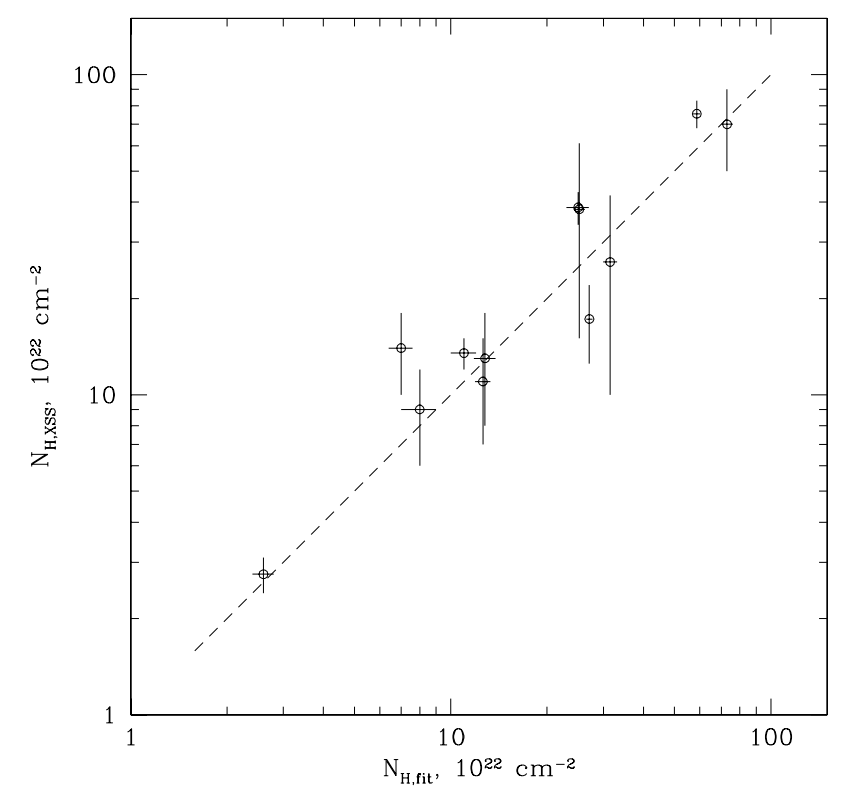

Fig. 2. Comparison of the absorption columns estimated from the XSS $8-20 \mathrm{keV} / 3-8 \mathrm{keV}$ count rate ratio, assuming a $\Gamma=1.8$ powerlaw intrinsic spectrum, with those derived through spectral analaysis of RXTE/PCA pointed observations for Seyfert 2 galaxies with a significant XSS detection of neutral absorption. The error bars are $1 \sigma$ statistical uncertainties.

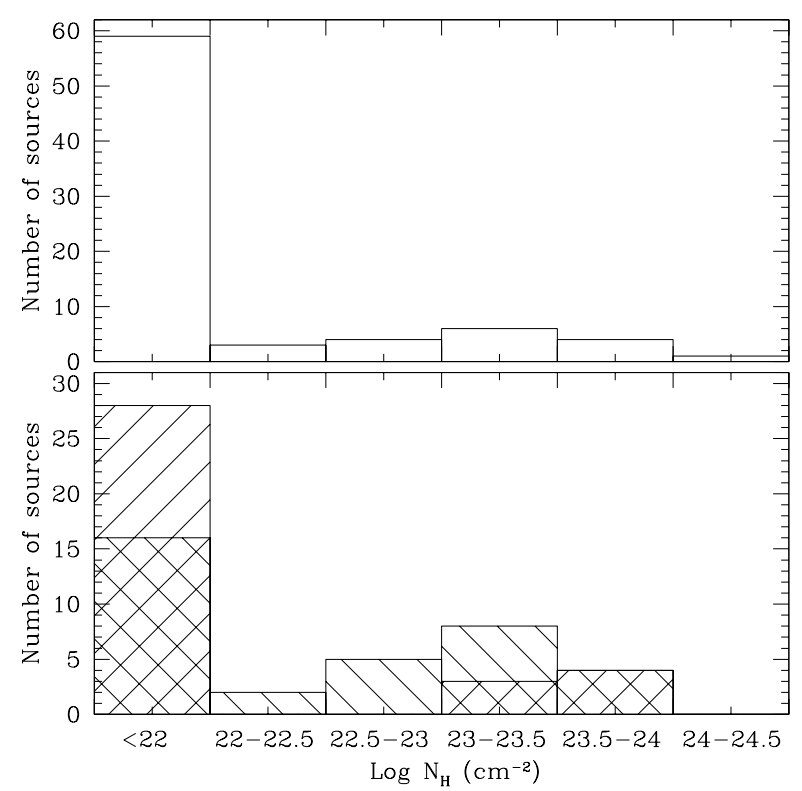

Fig. 3. Upper panel: observed distribution in intrinsic absorption column density $\left(N_{\mathrm{H}}\right)$ of emission-line AGNs in our sample. Lower panel: estimated $N_{\mathrm{H}}$ distribution of the unidentified XSS sources, AGN candidates. Two different histograms are presented, one computed by adopting $N_{\mathrm{H}}=0$ in those cases where the measured $N_{\mathrm{H}}$ value is smaller than the $1 \sigma$ statistical uncertainty, and the other computed using the best-fit $N_{\mathrm{H}}$ values, regardless of their errors.

In Fig. 3 we show the observed distribution of our nonblazar AGNs in $N_{\mathrm{H}}$. In obtaining this plot, we adopted $N_{\mathrm{H}}=0$ in the 11 cases where only an upper limit exceeding $10^{22} \mathrm{~cm}^{-2}$ is available. This is a reasonable assumption because all these sources are optically classified as type 1 AGNs. One can see 
that most of the sources in the sample have $N_{\mathrm{H}}<10^{22} \mathrm{~cm}^{-2}$. However, the remaining 18 AGNs provide us with enough information to reconstruct the physical $N_{\mathrm{H}}$ distribution of AGNs (see Sect. 3). As expected, almost all of our AGNs with $N_{\mathrm{H}}>$ $10^{22} \mathrm{~cm}^{-2}$ are optically classified as Seyfert 2 galaxies. One exception is NGC 4151, a well-known Seyfert 1 galaxy. We here point out another possible one - ESO 323-G077. Should our crude estimate (based on the hardness ratio) $N_{\mathrm{H}}=(6 \pm 3) \times$ $10^{23} \mathrm{~cm}^{-2}$ be confirmed by future X-ray spectroscopic observations, this source will present a unique example of a Seyfert 1 galaxy with a heavily absorbed X-ray spectrum. We note that our identification of this source in Paper I was based on its optical brightness ( $\left.m_{V}=13.2 \mathrm{mag}\right)$ and the suggestion by Schmid et al. (2003), motivated by spectropolarimetry of the object, that ESO 323-G077 may be a transition case between Seyfert 1 and Seyfert 2 galaxies due to its orientation.

\subsection{Unidentified sources}

There are 35 sources in the XSS catalog that remain unidentified (Table 2). We argued in Paper I that a large, probably dominant fraction of these sources are previously unknown AGNs. The main argument was that the distribution of their effective 3-20 keV spectral slopes is similar to that of the identified AGNs in the catalog. Being of great interest and waiting for identification, the unidentified sources present the largest source of incompleteness for the present statistical study. We note that a negligibly small number (4) of the unidentified sources have measured count rates falling below the $4 \sigma_{\text {conf }}$ confusion limit described above.

Assuming that the unidentified sources are AGNs and that their intrinsic spectrum is a power law with $\Gamma=1.8$, we can estimate intrinsic absorption column densities for them from the measured hardness ratios, exactly as we did before for the known AGNs. Since the candidate AGNs belong to the faint tail of the XSS catalog, their estimated aborption columns have large associated statistical uncertainties $\delta N_{\mathrm{H}}$, in fact in many cases only an upper limit can be given (see Table 2). We have therefore built two different $N_{\mathrm{H}}$ distributions, one by adopting $N_{\mathrm{H}}=0$ in those cases where $N_{\mathrm{H}}<\delta N_{\mathrm{H}}$, and another by using the best-fit $N_{\mathrm{H}}$ values in all cases. The true distribution likely lies between these two approximations. As shown in Fig. 3, if the unidentified sources are AGNs, they are similarly or somewhat more absorbed on average than our identified AGNs. We shall return to this point in Sect. 3. We point out that in the above analysis we assumed that $z=0$ for the unidentified sources, therefore the inferred column densities may be somewhat underestimated.

\subsection{Unresolved sources}

Our AGN sample may be additionally incomplete due to the presence in the XSS catalog of 12 sources associated with 2 or 3 unresolved astronomical objects including at least one AGN. Assuming that all sources in each of these groups contribute equally to the measured flux and applying the $4 \sigma$ detection criterion to the individual sources, we estimate that about
5 detectable AGNs are probably missing due to this manifestation of source confusion.

\subsection{Sample completeness}

It follows from the preceeding discussion that our input sample of 95 AGNs probably misses up to $\approx 41$ AGNs (including $4 \mathrm{C}+21.55$ with unknown redshift) meeting the XSS detection criterion, mostly as a result of the incomplete identification of the catalog. We can make allowance for this fact in the subsequent analysis (particularly when reconstructing the AGN luminosity function in Sect. 4) by introducing a completeness factor of $95 / 136 \approx 70 \%$, assuming that the luminosity distribution of unidentified AGNs is similar to that of the identified ones. The above value should be considered a lower limit because some of the unidentified sources are probably not AGNs. We may further introduce similar coefficients (lower limits) for the northern and southern hemispheres: $45 /(45+4+3) \approx 87 \%$ and $50 /(50+31+3) \approx 60 \%$. Our AGN sample is thus highly complete in the northern sky, and we shall take advantage of this fact later in the paper. Furthemore, our knowledge of the sample completeness is not limited by the above coefficients, in fact the crude information available on the X-ray spectra of the unidentified sources is utilized below in the investigation of the absorption distribution of AGNs.

\subsection{Comparison with the Piccinotti sample}

Our AGN sample includes 28 of the 35 sources composing the well-known HEAO-1/A2 sample of AGNs (Piccinotti et al. 1982; Malizia et al. 1999), based on a complete survey of the $|b|>20^{\circ}$ sky down to a limiting flux of $3.1 \times 10^{-11} \mathrm{erg} \mathrm{cm}^{-2} \mathrm{~s}^{-1}$ in the $2-10 \mathrm{keV}$ band. This degree of overlap of the two catalogs is consistent with our survey being more sensitive (for $90 \%$ of the sky at $|b|>10^{\circ}$ an equivalent $2-10 \mathrm{keV}$ sensitivity of $2 \times 10^{-11} \mathrm{erg} \mathrm{cm}^{-2} \mathrm{~s}^{-1}$ or better is achieved) combined with the fact that AGNs are known to be variable by a factor $\sim 2$ on a time scale of years.

\section{Distribution of AGNs in intrinsic absorption column density}

We now utilize the AGN sample defined in the preceeding section to study the distribution of local AGNs in intrinsic absorption column density, $f\left(N_{\mathrm{H}}\right)$. To this end, we employ the maximum likelihood technique, as described below. We note that calculations using the $1 / V_{\mathrm{m}}$ method lead to very similar results, but the maximum likelihood method allows us to estimate the errors of the output parameters more reliably, given the relatively small number of absorbed AGNs in our sample.

Assuming that $f\left(N_{\mathrm{H}}\right)$ is independent of the intrinsic AGN luminosity, we model $f\left(N_{\mathrm{H}}\right)$ in the range $N_{\mathrm{H}}<10^{24} \mathrm{~cm}^{-2}$ by a step-function

$f\left(N_{\mathrm{H}}\right)=f_{i}$ if $N_{\mathrm{H}, i}^{\min }<N_{\mathrm{H}}<N_{\mathrm{H}, i}^{\max }$,

where $i=1 \ldots 5, N_{\mathrm{H}, i}^{\min }=0,10^{22}, 10^{22.5}, 10^{23}, 10^{23.5} \mathrm{~cm}^{-2}$ and $N_{\mathrm{H}, i}^{\max }=10^{22}, 10^{22.5}, 10^{23}, 10^{23.5}, 10^{24} \mathrm{~cm}^{-2}$. By definition, 


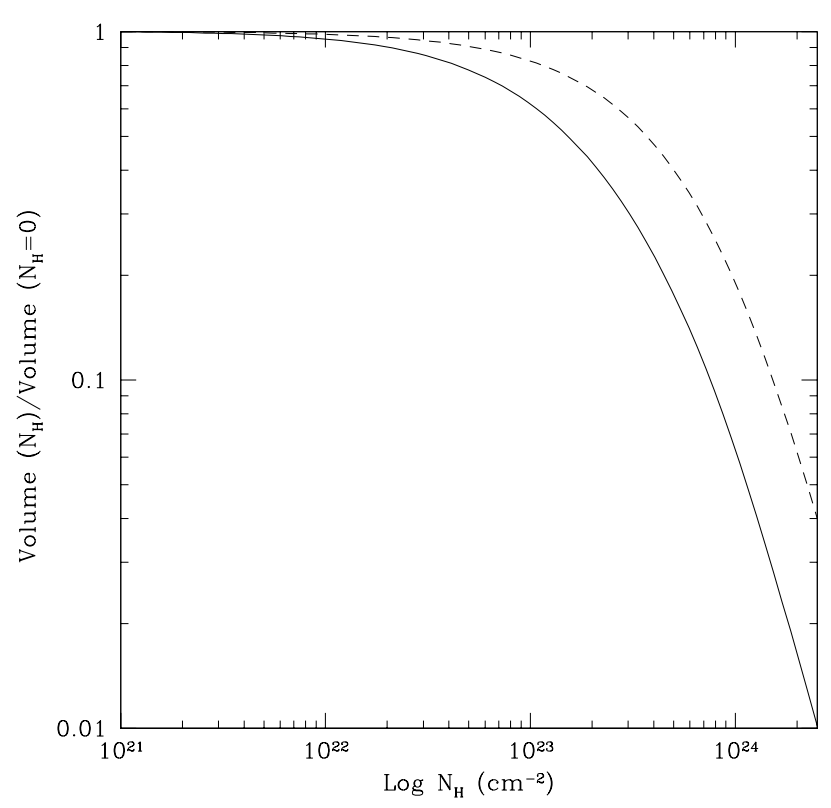

Fig. 4. Relative volume of space probed by the RXTE slew survey with respect to AGNs of given intrinsic (unabsorbed) luminosity with a $\Gamma=$ 1.8 power-law intrinsic spectrum as a function of absorption column density. The solid and dashed lines correspond to the $3-20 \mathrm{keV}$ and 8-20 keV bands, respectively.

$\sum_{i=1}^{5} f_{i}=1$, and therefore any 4 out of the $5 f$ s may be chosen as the free parameters.

We find the best-fit model by minimizing the maximum likelihood estimator, defined as follows:

$L=-2 \sum_{j} \ln \frac{f\left(N_{\mathrm{H}, j}\right) V_{\mathrm{m}}\left(L_{3-20, \text { int }, j}, N_{\mathrm{H}, j}\right)}{\int f\left(N_{\mathrm{H}}\right) V_{\mathrm{m}}\left(L_{3-20, \text { int }, j}, N_{\mathrm{H}}\right) \mathrm{d} N_{\mathrm{H}}}$,

where $j$ goes through each AGN in an input sample, and $V_{\mathrm{m}}\left(L_{3-20, \text { int }, j}, N_{\mathrm{H}}\right)$ is the space volume over which the $j$ th AGN with its intrinsic luminosity $L_{3-20 \text {,int }}$ could be detected by the survey if its spectrum were a $\Gamma=1.8$ power law absorbed by gas column $N_{\mathrm{H}}$. The integration in Eq. (2) is carried out from $N_{\mathrm{H}}=0$ to $10^{24} \mathrm{~cm}^{-2}$.

The relative survey volume, computed from the RXTE/PCA energy response and XSS exposure map (see Figs. 1-3 in Paper I), is shown as a function of $N_{\mathrm{H}}$ in Fig. 4. One can see that $V_{\mathrm{m}}$ begins to decrease noticeably above $N_{\mathrm{H}}=10^{23} \mathrm{~cm}^{-2}$ and drops 15 times by $10^{24} \mathrm{~cm}^{-2}$, as the low-energy cutoff in the X-ray spectrum reaches $\sim 10 \mathrm{keV}$. This causes the paucity of Compton thick sources in our catalog. Also shown in Fig. 4 is a similar plot obtained for the hard $(8-20 \mathrm{keV})$ subband, where the survey sensitivity is less affected by intrinsic absorption. We shall use the latter dependence in Sect. 4.4.

The parameter errors are estimated using a standard procedure: for each of the 4 fitted $f_{i}, 1 \sigma$ lower and upper limits are derived that correspond to an increase of $L$ by 1 from its minimum value while the other parameters are adjusted to minimize $L$. The confidence region for the 5 th (not used in the fitting) $f_{i}$ is obtained by repeating the procedure, considering this fraction a free parameter instead of one of the others.

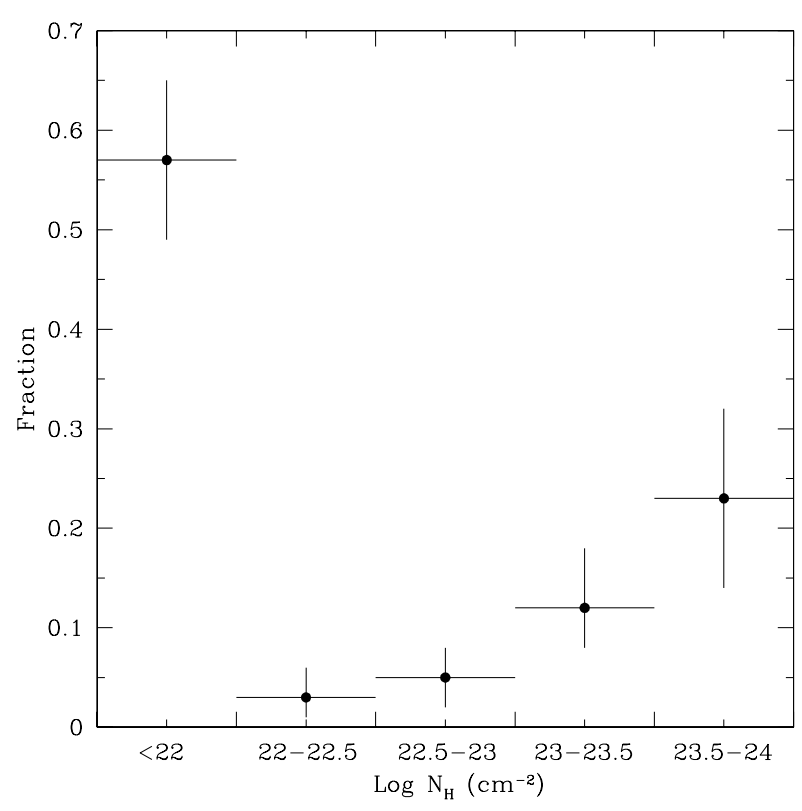

Fig. 5. Physical distribution in absorption column density of Comptonthin emission-line AGNs inferred from the XSS AGN sample. The error bars in this and subsequent figures represent $1 \sigma$ statistical uncertainties.

Performing an analysis along the above lines for our sample of 77 emission-line AGNs we obtain the $N_{\mathrm{H}}$ distribution shown in Fig. 5. We find that $57 \pm 8 \%$ of AGNs with $N_{\mathrm{H}}<10^{24} \mathrm{~cm}^{-2}$ are unabsorbed $\left(N_{\mathrm{H}}<10^{22} \mathrm{~cm}^{-2}\right)$ and also that more absorbed $\left(10^{23} \mathrm{~cm}^{-2}<N_{\mathrm{H}}<10^{24} \mathrm{~cm}^{-2}\right)$ sources are $4.1_{-1.6}^{+2.9}$ times as abundant as less absorbed $\left(10^{22} \mathrm{~cm}^{-2}<N_{\mathrm{H}}<10^{23} \mathrm{~cm}^{-2}\right)$ ones.

It is important to assess the effect of incompleteness of our AGN sample on the above result. As a first test, we compare in Fig. 6 the $N_{\mathrm{H}}$ distributions inferred from the northern and southern subsamples. These distributions are apparently similar and consistent with that obtained for the whole sample (Fig. 5), and we recall that our AGN sample is highly complete in the northern hemisphere. As a further test, we can build a $N_{\mathrm{H}}$ distribution for our unidentified sources by assuming that all of them are AGNs with an intrinsic $\Gamma=1.8$ power-law spectrum. By adopting that $N_{\mathrm{H}}=0$ if $N_{\mathrm{H}}<\delta N_{\mathrm{H}}$ and alternatively using the $N_{\mathrm{H}}$ values given in Table 2 in all cases, we obtain two distributions, shown in Fig. 6, that likely bound the true one. This distribution is also not significantly different from that derived for the known AGNs. We conclude that the effect of incompleteness on the distribution shown in Fig. 5 is small.

\subsection{Luminosity dependence}

We now wish to investigate whether the $N_{\mathrm{H}}$ distribution depends on luminosity. To this end, we repeat the above analysis separately for non-blazar AGNs with intrinsic luminosity $L_{3-20 \text {,int }}$ below and above $10^{43.5} \mathrm{erg} \mathrm{s}^{-1}$. The dividing luminosity is chosen such that the two resulting subsamples contain similar numbers of sources (37 and 40), which maximizes the statistical quality of the analysis. We obtain drastically different absorption distributions for the low-luminosity and high-luminosity subsamples, as shown in Fig. 7. While two 


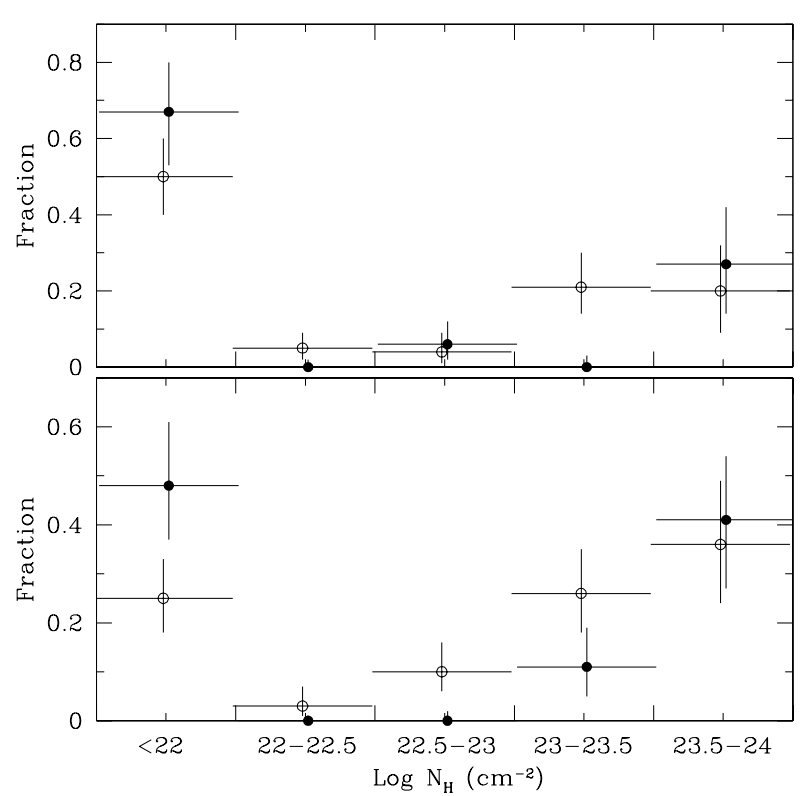

Fig. 6. Upper panel: AGN absorption column density distribution inferred from the northern (filled circles) and southern (open circles) subsamples of identified AGNs. Lower panel: $N_{\mathrm{H}}$ distribution estimated for the unidentified XSS sources, assuming that they are AGNs. One estimate (filled circles) is obtained by adopting $N_{\mathrm{H}}=0$ in those cases where the measured $N_{\mathrm{H}}$ value is smaller than the $1 \sigma$ statistical uncertainty, another one (open circles) is based on the measured $N_{\mathrm{H}}$ values. The different sets of data points are slightly shifted relative to each other along the horizontal axis for better visibility.

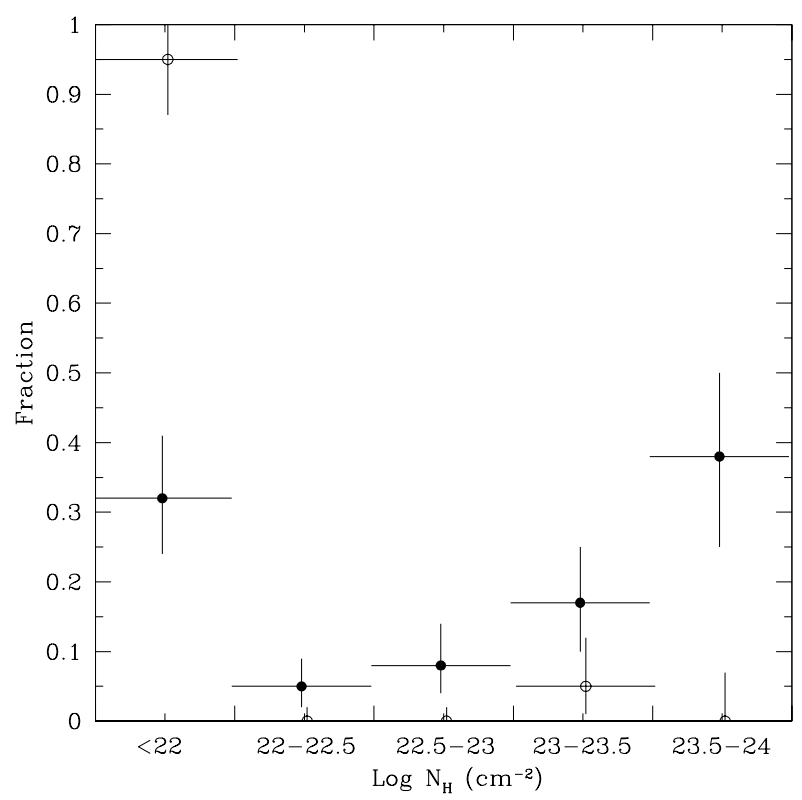

Fig. 7. Absorption column density distribution of emission-line AGNs with luminosity $L_{3-20}<10^{43.5} \mathrm{erg} \mathrm{s}^{-1}$ (filled circles) and $L_{3-20}>$ $10^{43.5} \mathrm{erg} \mathrm{s}^{-1}$ (open circles).

thirds of AGNs with $L_{3-20, \text { int }}<10^{43.5} \mathrm{erg} \mathrm{s}^{-1}$ are absorbed $\left(10^{22} \mathrm{~cm}^{-2}<N_{\mathrm{H}}<10^{24} \mathrm{~cm}^{-2}\right)$, the corresponding fraction among those with $L_{3-20 \text {,int }}>10^{43.5} \mathrm{erg} \mathrm{s}^{-1}$ is less than $20 \%$ ( $2 \sigma$ upper limit). We emphasize once again that we do not consider Compton thick sources. We also note that since the difference between observed and intrinsic luminosity is fairly small, $\log \left(L_{3-20, \text { int }} / L_{3-20}\right)=0.1(0.5)$ for $N_{\mathrm{H}}=10^{23}\left(10^{24}\right) \mathrm{cm}^{-2}$, the above result will essentially not change if the division of sources at $10^{43.5} \mathrm{erg} \mathrm{s}^{-1}$ is done in terms of the observed luminosity.

We point out that the tight upper limit obtained above on the fraction of absorbed high-luminosity AGNs is linked to the fact that there is only one source (Seyfert 2 galaxy NGC 7582) with $L_{3-20 \text {,int }}>10^{43.5} \mathrm{erg} \mathrm{s}^{-1}$ and $N_{\mathrm{H}}>10^{22} \mathrm{~cm}^{-2}$ in our sample. We recall however that we simply assumed $N_{\mathrm{H}}=0$ for those 11 sources (all are optically type 1s) for which only an upper limit on $N_{\mathrm{H}}$ exceeding $10^{22} \mathrm{~cm}^{-2}$ is available from observations. Seven of these sources have $L_{3-20, \text { int }}>10^{43.5} \mathrm{erg} \mathrm{s}^{-1}$. There is a non-negligible probability that 1 or 2 of these sources are absorbed with $N_{\mathrm{H}}>10^{22} \mathrm{~cm}^{-2}$. However, even then the fraction of absorbed AGNs with $L_{3-20 \text {,int }}>10^{43.5} \mathrm{erg} \mathrm{s}^{-1}$ will remain small: $10 \pm 8 \%$ and $14 \pm 7 \%$, respectively.

\subsection{Comparison with other studies}

Until recently, our knowledge of the distribution of AGNs in intrinsic absorption was restricted to the local Universe and originated from optically selected samples. In particular, it was known that Seyfert 2 galaxies outnumber their Seyfert 1 counterparts by a factor of 4 (Maiolino \& Rieke 1995). Also, Risaliti et al. (1999) estimated the column density distribution of Seyfert $2 \mathrm{~s}$ using a sample limited in the intensity of the [O iii] $5007 \AA$ narrow emission line taken as an indicator (after some correction) of the intrinsic AGN flux. These authors concluded that Compton thick $\left(N_{\mathrm{H}}>10^{24} \mathrm{~cm}^{-2}\right)$ and Compton thin $\left(N_{\mathrm{H}}<10^{24} \mathrm{~cm}^{-2}\right)$ Seyfert 2 s are approximately equally abundant. Since only a small fraction of Seyfert $1 \mathrm{~s}$ exhibit absorbed X-ray spectra, the above papers imply a 2:1 ratio of Compton thin absorbed $\left(10^{22} \mathrm{~cm}^{-2}<N_{\mathrm{H}}<10^{24} \mathrm{~cm}^{-2}\right)$ to unabsorbed $\left(N_{\mathrm{H}}<10^{22} \mathrm{~cm}^{-2}\right)$ AGNs. This is in good agreement with our result for low-luminosity $\left(L_{3-20}<10^{43.5} \mathrm{erg} \mathrm{s}^{-1}\right)$ AGNs. However, our result for the ratio of strongly absorbed $\left(10^{23} \mathrm{~cm}^{-2}<N_{\mathrm{H}}<10^{24} \mathrm{~cm}^{-2}\right)$ to moderately absorbed $\left(10^{22} \mathrm{~cm}^{-2}<N_{\mathrm{H}}<10^{23} \mathrm{~cm}^{-2}\right)$ low-luminosity AGNs of $4.1_{-1.6}^{+2.9}$ differs from that of Risaliti et al. of $1.7 \pm 0.7$ (the errors are $1 \sigma$ ), but not significantly. Since the Risaliti et al. sample consists almost entirely of AGNs of low luminosity, the completely different $N_{\mathrm{H}}$ distribution that we infer for AGNs with $L_{3-20}>10^{43.5} \mathrm{erg} \mathrm{s}^{-1}$ could not be assessed on its basis.

More recently, medium-sensitivity and deep X-ray surveys have begun to provide statistical information on the $N_{\mathrm{H}}$ distribution of AGNs. In particular, utilizing a sample constructed from several surveys performed in the standard 2-10 keV band with HEAO-1, ASCA and Chandra, Ueda et al. (2003) came to the same conclusion that we reach here that the fraction of absorbed (Compton thin) AGNs decreases with luminosity. Furthermore, the fraction of absorbed sources among lowluminosity $\left(L_{3-20} \lesssim 10^{43.5} \mathrm{erg} \mathrm{s}^{-1}\right.$ ) AGNs found by these authors $(\approx 60 \%)$ is in good agreement with our estimate $(68 \pm$ $8 \%$ ). On the other hand, their estimated value of $\sim 30-40 \%$ for this fraction among higher-luminosity AGNs is only marginally consistent with our $2 \sigma$ upper limit of $20 \%$ (possibly $30 \%$ if 1 or 2 of the 7 type 1 AGNs in our sample with upper limits 
on the column density have $N_{\mathrm{H}}>10^{22} \mathrm{~cm}^{-2}$, see Sect. 3.1). However, the results of Ueda et al. quoted above are obtained for a heterogeneous sample combining local and very distant AGNs $(z=0.01-3)$, while our estimates are made for the local AGN population. The apparent discrepancy may therefore hint at a substantial cosmological evolution of the intrinsic absorption distribution of powerful, quasar-like AGNs.

In another work, Steffen et al. (2003) investigated the fraction of optically identified broad-line AGNs among X-ray sources detected at $2-8 \mathrm{keV}$ with Chandra and ASCA. For the $z=0.1-1$ population, this fraction was found to increase with luminosity, from less than $50 \%$ at $L_{3-20} \lesssim 10^{43} \mathrm{erg} \mathrm{s}^{-1}$ to more than $85 \%$ at $L_{3-20} \gtrsim 10^{44} \mathrm{erg} \mathrm{s}^{-1}$, with most of the uncertainty resulting from the large number of unidentified sources, especially at low luminosity. If we associate broad-line sources with X-ray unabsorbed AGNs, the Steffen et al. results for the $z=0.1-1$ population appear to be consistent with ours for the local one.

\section{X-ray AGN luminosity function}

We now address the X-ray luminosity function of nearby AGNs. We define this function $\phi\left(L_{3-20}\right)$ as the number density of AGNs per $\log L_{3-20}$, where $L_{3-20}$ is the observed luminosity in the 3-20 keV range.

We first estimate $\phi\left(L_{3-20}\right)$ in binned form using the conventional $1 / V_{\mathrm{m}}$ method (Schmidt 1968). Here, as in the previous section, $V_{\mathrm{m}}\left(L_{3-20}, N_{\mathrm{H}}\right)$ is the space volume over which a given AGN with its observed luminosity $L_{3-20}$ and estimated absorption column $N_{\mathrm{H}}$ (again assuming an intrinsic power-law spectrum with $\Gamma=1.8$ ) could be detected.

As a next step, we approximate the data by a smoothly connected two power-law model

$\phi\left(L_{3-20}\right)=\frac{A}{\left(L_{3-20} / L_{*}\right)^{\gamma_{1}}+\left(L_{3-20} / L_{*}\right)^{\gamma_{2}}}$.

For this purpose, the maximum likelihood estimator

$L=-2 \sum_{j} \ln \frac{\phi\left(L_{3-20, j}\right) V_{0}\left(L_{3-20, j}\right)}{\int \phi\left(L_{3-20}\right) V_{0}\left(L_{3-20}\right) \mathrm{d} \log L_{3-20}}$

is used, where $j$ goes over all sampled AGNs.

The sampled volume $V_{0}\left(L_{3-20}\right)$ introduced above derives from the previously defined $V_{\mathrm{m}}\left(L_{3-20}, N_{\mathrm{H}}\right)$ as follows:

$$
\begin{aligned}
V_{0}\left(L_{3-20}\right)= & \int f\left(N_{\mathrm{H}}\right) V_{\mathrm{m}}\left(L_{3-20}, N_{\mathrm{H}}\right) \mathrm{d} N_{\mathrm{H}} \\
= & V_{\mathrm{m}}\left(L_{3-20}, N_{\mathrm{H}}=0\right) \\
& \times \begin{cases}0.78, & L_{3-20}<10^{43.5} \mathrm{erg} \mathrm{s}^{-1} \\
1, & L_{3-20}>10^{43.5} \mathrm{erg} \mathrm{s}^{-1} .\end{cases}
\end{aligned}
$$

According to this formulation, the $N_{\mathrm{H}}$ values estimated for the individual AGNs in our sample are not taken into account, but allowance is made for the average absorption distribution of AGNs $f\left(N_{\mathrm{H}}\right)$ as a function of luminosity derived in Sect. 3. It means that we evaluate the probability of observing an AGN with a given luminosity $L_{3-20}$, regardless of its intrinsic absorption. We point out that the binned luminosity function obtained

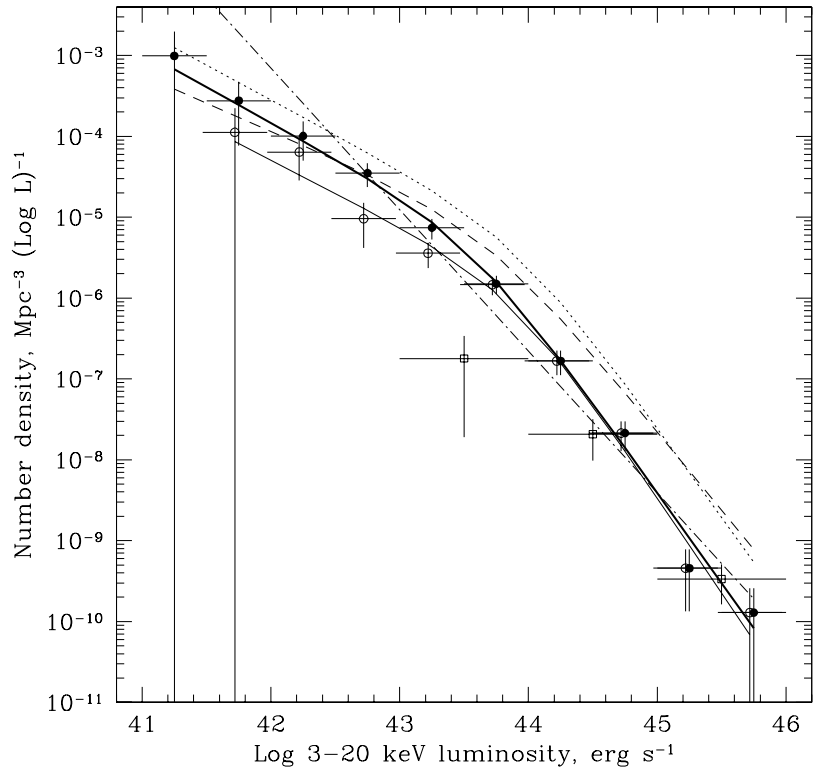

Fig. 8. Local 3-20 keV luminosity functions obtained here for (i) Compton-thin $\left(N_{\mathrm{H}}<10^{24} \mathrm{~cm}^{-2}\right)$ non-blazar AGNs: in binned form (solid circles with $1 \sigma$ error bars) and an analytic approximation (thick solid curve) given by Eq. (3) with parameter values quoted in Table 3, (ii) similarly for unabsorbed $\left(N_{\mathrm{H}}<10^{22} \mathrm{~cm}^{-2}\right)$ non-blazar AGNs (open circles and thin solid curve), and (iii) for blazars (open squares within wider bins, no analytic fit is presented). The second set of data points is slighlty shifted along the luminosity axis for better visibility. For comparison are presented previous estimates of the local 2-10 keV AGN luminosity function, recomputed here to the $3-20 \mathrm{keV}$ band and to our adopted value $H_{0}=75 \mathrm{~km} \mathrm{~s}^{-1} \mathrm{Mpc}^{-1}$ : by Piccinotti et al. (1982) (dash-dotted curve), by Ueda et al. (2003) (for Compton-thin sources, dotted curve) and by La Franca et al. (2002) (for unabsorbed sources, dashed curve).

using the $1 / V_{\mathrm{m}}$ method inherently takes into account individual column densities.

Minimizing $L$ yields the best-fit values of the break luminosity $L_{*}$ as well as of the slopes $\gamma_{1}$ and $\gamma_{2}$. The fitting procedure does not however allow us to determine the normalizing constant $A$ of the best-fit model. We thus calculate $A$ from the condition that the number of AGNs predicted by the model is equal to the actual number of AGNs in our sample.

We perform calculations for the luminosity range $10^{41} \mathrm{erg} \mathrm{s}^{-1}<L_{3-20}<10^{46} \mathrm{erg} \mathrm{s}^{-1}$, which includes all of our emission-line AGNs except NGC 4945. The estimated luminosity of NGC 4945 is $8 \times 10^{40} \mathrm{erg} \mathrm{s}^{-1}$ and as was noted in Sect. 2, this is the only Compton thick AGN in our sample while the subject of our study is Compton thin AGNs. We present the derived binned luminosity function and bestfit model in Fig. 8. The parameters of the model together with their estimated $1 \sigma$ statistical uncertainties are summarized in Table 3 . The normalization $A$ is given without an error because this parameter is strongly correlated with the others. The analytic fit is apparently in good agreement with the binned $\phi\left(L_{3-20}\right)$; the good quality of the fit is confirmed by the Kolmogorov-Smirnov test.

The RXTE slew survey is effectively limited by a redshift $z=0.1$ for AGNs with $L_{3-20}<10^{44.5} \mathrm{erg} \mathrm{s}^{-1}$. However, 
Table 3. 3-20 keV AGN luminosity function parameters.

\begin{tabular}{|c|c|c|c|c|c|c|c|c|c|}
\hline Sample & $\begin{array}{c}\log L_{3-20} \\
\mathrm{erg} \mathrm{s}^{-1}\end{array}$ & Size & $\begin{array}{l}\log L_{*}{ }^{a} \\
\mathrm{erg} \mathrm{s}^{-1}\end{array}$ & $\gamma_{1}$ & $\gamma_{2}$ & $\begin{array}{c}A^{b} \\
\mathrm{Mpc}^{-3}\end{array}$ & $\begin{array}{c}W_{3-20}(>41) \\
10^{38} \mathrm{erg} \mathrm{s}^{-1} \mathrm{Mpc}^{-3}\end{array}$ & $\begin{array}{l}N_{3-20}(>41) \\
10^{-4} \mathrm{Mpc}^{-3}\end{array}$ & $P_{\mathrm{KS}}^{c}$ \\
\hline Total & $41-46$ & 76 & $43.58_{-0.30}^{+0.32}$ & $0.88_{-0.20}^{+0.18}$ & $2.24_{-0.18}^{+0.22}$ & $6.0(8.6) \times 10^{-6}$ & $4.3_{-0.6}^{+0.7}\left(6.1_{-0.9}^{+1.0}\right)$ & $5_{-2}^{+4}\left(7_{-3}^{+6}\right)$ & $>0.9$ \\
\hline Total & $41-45$ & 73 & $43.52_{-0.40}^{+0.40}$ & $0.86_{-0.24}^{+0.20}$ & $2.16_{-0.26}^{+0.36}$ & $7.3 \times 10^{-6}$ & $4.3_{-0.6}^{+0.7}$ & $5_{-2}^{+4}$ & $>0.9$ \\
\hline Unabsorbed & $41-46$ & 59 & $43.84_{-0.26}^{+0.26}$ & $0.74_{-0.20}^{+0.20}$ & $2.32_{-0.22}^{+0.26}$ & $1.9 \times 10^{-6}$ & $1.9_{-0.3}^{+0.4}$ & $1.4_{-0.6}^{+1.5}$ & $>0.9$ \\
\hline North & $41-45$ & 33 & $43.78_{-0.40}^{+0.44}$ & $0.96_{-0.24}^{+0.20}$ & $2.26_{-0.26}^{+0.34}$ & $2.5(2.9) \times 10^{-6}$ & $3.8_{-1.0}^{+1.1}\left(4.4_{-1.2}^{+1.3}\right)$ & $5_{-2}^{+5}\left(6_{-2}^{+6}\right)$ & 0.88 \\
\hline South & $41-46$ & 43 & $43.24_{-0.42}^{+0.50}$ & $0.66_{-0.48}^{+0.34}$ & $2.14_{-0.22}^{+0.32}$ & $2.2(3.7) \times 10^{-5}$ & $4.5_{-0.8}^{+1.0}\left(7.5_{-1.3}^{+1.7}\right)$ & $4_{-2}^{+4}\left(7_{-3}^{+7}\right)$ & $>0.9$ \\
\hline
\end{tabular}

${ }^{a}$ All presented uncertainties are $1 \sigma$.

${ }^{b}$ In parentheses are given values corrected for maximum possible sample incompleteness.

${ }^{c}$ Kolmogorov-Smirnov probability.

our sample of emission-line AGNs includes 6 sources located at $z=0.1-0.3$, all of which have $L_{3-20}>10^{44.7} \mathrm{erg} \mathrm{s}^{-1}$. Nevertheless, we are confident that the obtained luminosity function is characteristic of the local Universe at $z<0.1$. To prove this, we repeat the fitting in the narrower luminosity range $10^{41} \mathrm{erg} \mathrm{s}^{-1}<L_{3-20}<10^{45} \mathrm{erg} \mathrm{s}^{-1}$, so that the fraction of AGNs with $z>0.1$ reduces to 3 out of 7 in the interval $10^{44.5} \mathrm{erg} \mathrm{s}^{-1}<L_{3-20}<10^{45} \mathrm{erg} \mathrm{s}^{-1}$. As can be seen in Table 3 , the best-fit model remains essentially unchanged.

We also present in Fig. 8 and Table 3 the luminosity function obtained for unabsorbed $\left(N_{\mathrm{H}}<10^{22} \mathrm{~cm}^{-2}\right)$ emission-line AGNs. In calculating the best-fit model in this case, the volume correction given by Eq. (5) was not made. As could have been expected from the behavior of the $N_{\mathrm{H}}$ distribution with luminosity (Sect. 3), the contribution of unabsorbed sources to the number density of Compton thin AGNs is smaller than 50\% at $L_{3-20}<L_{*} \approx 10^{43.5} \mathrm{erg} \mathrm{s}^{-1}$, but becomes dominant at $L_{3-20}>$ $L_{*}$. It is interesting that the dramatic change in the intrinsic absorption distribution with luminosity that we discussed in Sect. 3 seems to take place somewhere near the break of the AGN luminosity function, although our sample is not large enough to follow in detail the absorption distribution as a function of luminosity.

We have further estimated the X-ray luminosity function of blazars, disregarding the fact that only the 9 lower-luminosity $\left(L_{3-20}<10^{45} \mathrm{erg} \mathrm{s}^{-1}\right)$ blazars in our sample belong to the local population $(z \lesssim 0.1$ ) of AGNs. Interestingly, as demonstrated by Fig. 8, the number density of blazars becomes comparable to that of normal (emission-line) AGNs at observed luminosities above $\sim 10^{44} \mathrm{erg} \mathrm{s}^{-1}$. It is worth noting that our blazars, selected in the 3-20 keV band, have spectra (see Paper I) characterized by a broad distribution of slopes $(\Gamma \sim 1-3)$ centered near the canonical $\Gamma=1.8$ value for unabsorbed emission-line AGNs.

\subsection{Volume emissivity}

Multiplying the luminosity functions shown in Fig. 8 by the luminosity yields a new plot (Fig. 9) that allows one to compare the contributions of AGNs with various luminosities to the local X-ray volume emissivity. We can see that AGNs, mostly absorbed ones, with luminosities in the range $10^{41}-10^{43.5} \mathrm{erg} \mathrm{s}^{-1}$

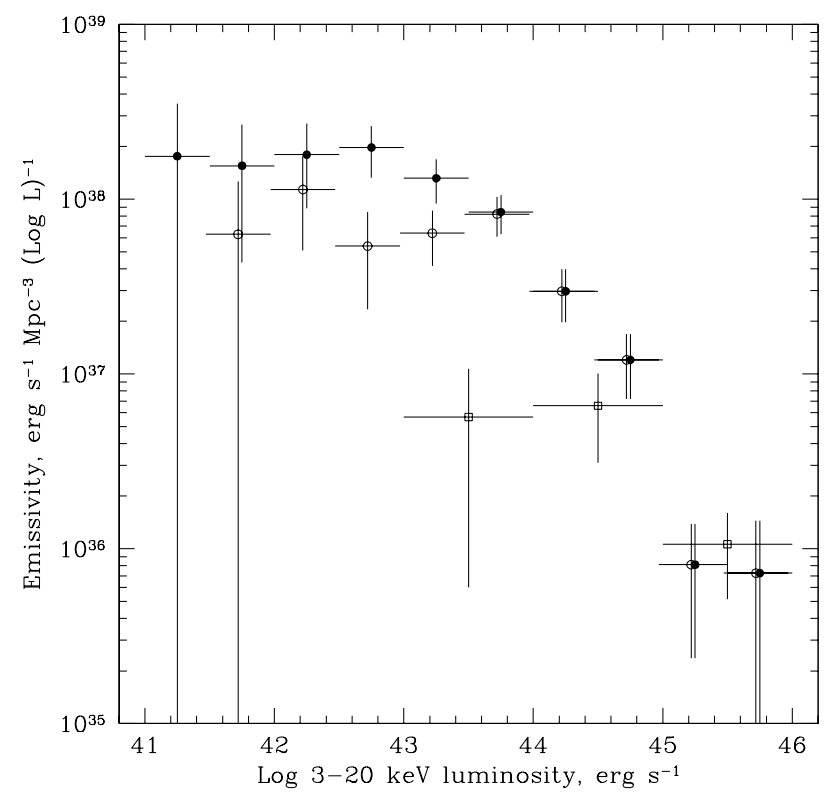

Fig. 9. Contribution of AGNs with various luminosities to the local 3-20 keV volume emissivity, estimated from the binned luminosity functions presented in Fig. 8: of Compton-thin non-blazar AGNs (filled circles), of unabsorbed non-blazar AGNs (open circles), and of blazars (open squares).

release similar amounts of energy in X-rays per $\log L_{3-20}$. We can integrate once more over luminosity,

$W_{3-20}(>41)=\int_{41}^{\infty} \phi\left(L_{3-20}\right) L_{3-20} \mathrm{~d} \log L_{3-20}$,

to estimate the cumulative emissivity of emission-line AGNs with $L_{3-20}>10^{41} \mathrm{erg} \mathrm{s}^{-1}$. The resulting value is presented in Table 3 together with a $1 \sigma$ statistical uncertainty determined by exploring the likelihood distribution over the parameter space. It can be seen that $W_{3-20}(>41)$ is well constrained by the data.

For some applications, the total number density of AGNs with $L_{3-20}>10^{41} \mathrm{erg} \mathrm{s}^{-1}$,

$N_{3-20}(>41)=\int_{41}^{\infty} \phi\left(L_{3-20}\right) \mathrm{d} \log L_{3-20}$,

also might be important. Our estimate for this quantity is presented in Table 3 . We point out that the quoted number density 


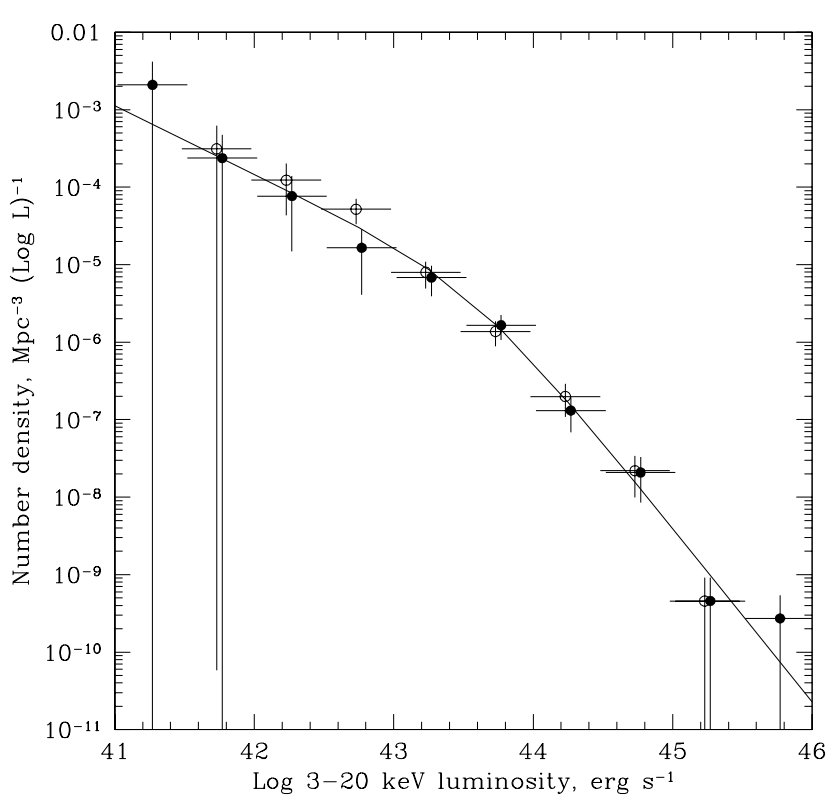

Fig. 10. 3-20 keV luminosity functions of local Compton-thin nonblazar AGNs inferred from the southern subsample (filled circles) and northern subsample (open circles), in comparison with the best-fit model (Eq. (3), Table 3) found for the whole AGN sample (solid line).

is dominated by AGNs located near the lower end of the sampled luminosity range and may increase by a substantial factor if AGNs with $L_{3-20}<10^{41} \mathrm{erg} \mathrm{s}^{-1}$ are counted.

\subsection{Additional checks of the results}

The 3-20 keV AGN luminosity function obtained above may be affected by both the incompleteness of the input sample and the inhomogeneous distribution of AGNs resulting from the local large scale structure. To address the first of these issues, we assumed that AGNs meeting the XSS detection criterion and not appearing in our sample have the same distribution in luminosity as the AGNs composing the sample. We can then estimate the maximum possible effect of sample incompleteness by correcting the best-fit model amplitude $A$ as well as the inferred integral quantitites $W_{3-20}(>41)$ and $N_{3-20}(>41)$ using the completeness coefficient defined in Sect. 2.4. The corrected amplitude is given in Table 3. The true amplitude of the luminosity function should lie somewhere between the uncorrected and corrected values.

In order to estimate the possible effect of the large scale structure, we have computed luminosity functions for our northern and southern subsamples of AGNs. The obtained binned distributions and corresponding best-fit analytic models are presented in Fig. 10 and Table 3. In addition, the amplitudes corrected for the incompleteness of both samples are given. The Kolmogorov-Smirnov test demonstrates that the two AGN subsamples could well be drawn from the same luminosity distribution: $P_{\mathrm{KS}}=0.35$. Furthermore, the estimated cumulative emissivity $W_{3-20}(>41)$ and number density $N_{3-20}(>41)$ are not significantly different for the northern and southern samples either.

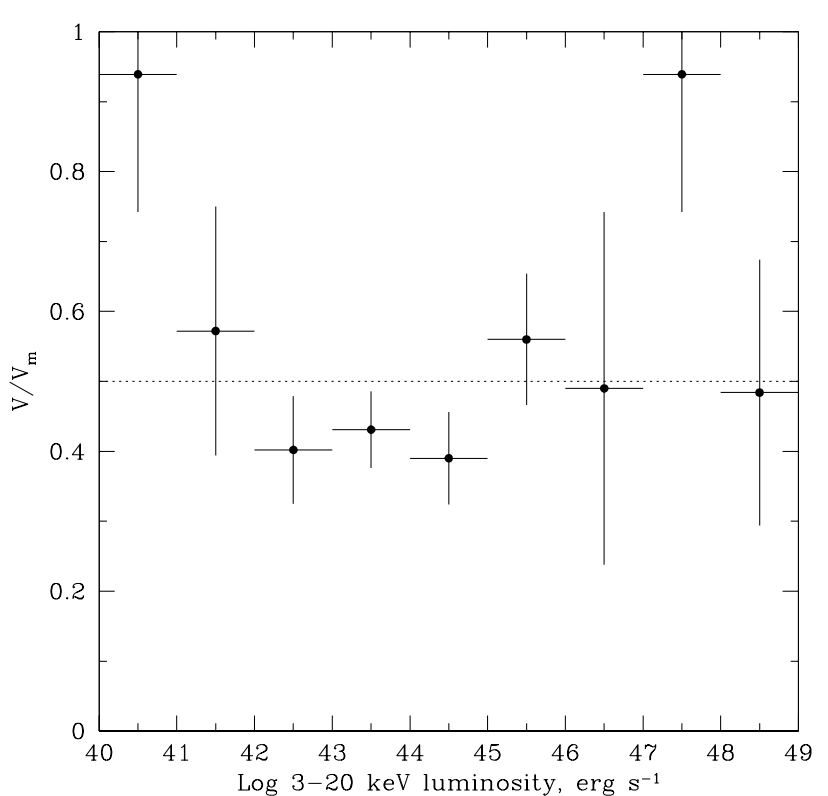

Fig. 11. $V / V_{\mathrm{m}}$ ratio averaged over luminosity bins for the whole XSS AGN sample. The error bars represent $1 \sigma$ statistical uncertainties.

Finally, we perform a standard $V / V_{\mathrm{m}}$ test (Schmidt 1968) for our AGN sample. To this end, a $V / V_{\mathrm{m}}$ ratio is calculated for each AGN as $(C / 4 \delta C)^{-3 / 2}$, where $C$ is the measured count rate in the $3-20 \mathrm{keV}$ band and $\delta C$ is the associated $1 \sigma$ statistical uncertainty. The factor 4 reflects the detection criterion defining the AGN sample. Then, averaging is performed in specified luminosity bins. As can be seen from Fig. 11, the observed deviations of the $V / V_{\mathrm{m}}$ ratio from the value 0.5 expected for a homogeneous distribution of sources are not statistically significant. In particular, averaging over the whole sample of emission-line AGNs gives $\left\langle V / V_{\mathrm{m}}\right\rangle=0.44 \pm 0.04$, which represents an insignificant $(1.5 \sigma)$ deviation from 0.5 .

We conclude that the obtained luminosity function is not significantly affected by the inhomogeneity of matter distribution in the local Universe. The possible incompleteness of our sample introduces a systematic uncertainty of $\sim 20 \%$ on the amplitude of the luminosity function, characterized by $W_{3-20}(>41)$, in addition to the statistical uncertainty of $\sim 15 \%$.

\subsection{Comparison with other studies}

The 3-20 keV AGN luminosity function obtained in this work surpasses in quality previously published luminosity functions obtained at photon energies above $2 \mathrm{keV}$ for the local $(z<0.1)$ AGN population. In Fig. 8, we show for comparison the $2-10 \mathrm{keV}$ luminosity function derived by Piccinotti et al. (1982) from their HEAO-1/A2 sample of AGNs (mostly unabsorbed ones). The small luminosity correction $\log \left(L_{3-20} / L_{2-10}\right)=0.1$ has a negligible effect on the comparison. Since the Piccinotti sample is relatively small ( $\sim 40 \%$ of ours) and covers a relatively narrow luminosity range $10^{42} \mathrm{erg} \mathrm{s}^{-1}<L_{2-10}<10^{45} \mathrm{erg} \mathrm{s}^{-1}$, the data could be well fitted by a power-law model with an index 1.75 . Within the 
uncertainties, the Piccinotti et al. luminosity function is in very good agreement with the one derived here.

In addition, much effort has been invested into studying the evolution of the 2-10 keV AGN luminosity function with redshift. In particular, La Franca et al. (2002) and Ueda et al. (2003) have constructed large samples of AGNs for this purpose. The former includes 158 optically type 1 AGNs selected from HEAO-1, BeppoSAX and ASCA surveys, and the latter consists of 247 AGNs detected by HEAO-1, ASCA and Chandra, including a substantial number of X-ray absorbed sources. In these works, both the shape of the luminosity function and its evolution out to high redshift are fitted simultaneously to the observed distribution of AGNs on the luminosityredshift plane. It is interesting to compare the present-day luminosity functions predicted by these studies with the one directly determined here.

Ueda et al. (2003) present their results in terms of the intrinsic $2-10 \mathrm{keV}$ luminosity $L_{2-10}$,int. Although our luminosity function is defined in terms of the observed $3-20 \mathrm{keV}$ luminosity $L_{3-20}$, it will hardly change after recalculation in terms of $L_{3-20, \text { int }}$ since $\left\langle\log \left(L_{3-20, \text { int }} / L_{3-20}\right)\right\rangle=0.1$ (0) for the observed $N_{\mathrm{H}}$ distribution of AGNs with $L_{3-20}<$ $10^{43.5} \mathrm{erg} \mathrm{s}^{-1}\left(L_{3-20}>10^{43.5} \mathrm{erg} \mathrm{s}^{-1}\right)$. The similarly small $\log \left(L_{3-20, \text { int }} / L_{2-10 \text {,int }}\right)=0.1$ correction counteracts the previous one at low luminosity. We can therefore take approximately $\log \left(L_{3-20} / L_{2-10, \text { int }}\right)=0.05$ for the entire luminosity range. On the other hand, the luminosity function of La Franca et al. (2002) is obtained for unabsorbed AGNs and should thus be compared with our corresponding result for this case (making a small correction $\left.\log \left(L_{3-20} / L_{2-10}\right)=0.1\right)$.

The comparison is done in Fig. 8, and it can be seen that the amplitude of our luminosity functions derived for all AGNs and for unabsorbed ones is smaller by at least a factor of 2 than predicted for $z=0$ by Ueda et al. (2003) and La Franca et al. (2002), respectively. Part of this apparent discrepancy (less than a factor of 1.4 and this is already reflected in Table 3 ) may result from the possible incompleteness of our sample. It is difficult to make a formal statement as to whether the remaining difference can be accounted for by the statistical uncertainties. Despite the different amplitudes, the shapes of our luminosity function and those of Ueda et al. (2003) and La Franca et al. (2002) are in satisfactory agreement.

\subsection{8-20 keV luminosity function}

Our preceeding analysis was based on a sample of AGNs selected by the flux in the $3-20 \mathrm{keV}$ band. At the same time, we know the source fluxes in the subbands $3-8 \mathrm{keV}$ and 8-20 keV. In addition, as explained in Paper I, the sensitivity of the RXTE slew survey as a function of photon energy (determined by the energy response of the PCA instrument and by the background) is such that Compton thin AGNs $\left(N_{\mathrm{H}}<\right.$ $10^{24} \mathrm{~cm}^{-2}$ ) detectable at $8-20 \mathrm{keV}$ are always also detectable in the broader band 3-20 keV. We can therefore define a sample of AGNs selected in the 8-20 keV band from our 3-20 keV sample (Table 1 ) by applying the condition $C_{8-20} / \delta C_{8-20}>4$,

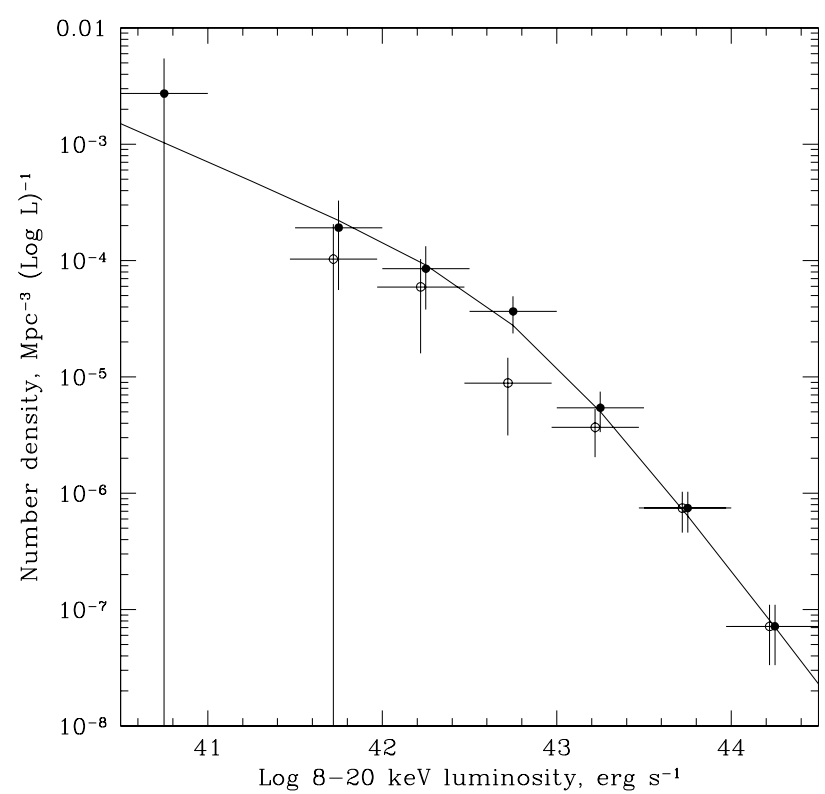

Fig. 12. Local $8-20 \mathrm{keV}$ luminosity functions obtained here for Compton-thin $\left(N_{\mathrm{H}}<10^{24} \mathrm{~cm}^{-2}\right)$ non-blazar AGNs: in binned form (solid circles with $1 \sigma$ error bars) and an analytic approximation (thick solid curve) given by Eq. (3) with parameter values quoted in Table 3, and for unabsorbed $\left(N_{\mathrm{H}}<10^{22} \mathrm{~cm}^{-2}\right)$ non-blazar AGNs (open circles, no analytic fit is presented).

where $C_{8-20}$ and $\delta C_{8-20}$ are the count rate and $1 \sigma$ uncertainty for the hard subband.

The hard X-ray selected sample consists of 45 AGNs, including 37 emission-line AGNs (24 unabsorbed and 13 absorbed) and 8 blazars. Among the 35 unidentified sources (Table 2) only 7 are detected in the $8-20 \mathrm{keV}$ band, and therefore the new AGN sample is at least $87 \%$ complete.

Using the above sample, we have built a 8-20 keV AGN luminosity function in the sampled range $10^{40.5} \mathrm{erg} \mathrm{s}^{-1}>L_{8-20}<$ $10^{44.5} \mathrm{erg} \mathrm{s}^{-1}$ (here $L_{8-20}$ is the observed $8-20 \mathrm{keV}$ luminosity) by the methods described earlier in this section. The results are presented in Fig. 12 and Table 4. In calculating the bestfit model we made a small volume correction to take into account the difference in the $N_{\mathrm{H}}$ distribution of low-luminosity and high-luminosity AGNs:

$$
\begin{aligned}
V_{0}\left(L_{8-20}\right)= & V_{\mathrm{m}}\left(L_{8-20}, N_{\mathrm{H}}=0\right) \\
& \times \begin{cases}0.84, & L_{8-20}<10^{43.2} \mathrm{erg} \mathrm{s}^{-1} \\
1, & L_{8-20}>10^{43.2} \mathrm{erg} \mathrm{s}^{-1} .\end{cases}
\end{aligned}
$$

This expression differs from Eq. (5) because the dependence of the survey volume on $N_{\mathrm{H}}$ is different for the $3-20 \mathrm{keV}$ and 8-20 keV bands (see Fig. 4 ), and since $\log \left(L_{3-20} / L_{8-20}\right)=0.3$ for $N_{\mathrm{H}}=0$ (for comparison, $\left\langle\log \left(L_{3-20} / L_{8-20}\right)\right\rangle=0.2$ for an ensemble of AGNs with $L_{3-20}<10^{43.5} \mathrm{erg} \mathrm{s}^{-1}$ and the derived $N_{\mathrm{H}}$ distribution).

The small size of our 8-20 keV selected sample compared to the 3-20 keV selected one leads to relatively large uncertainty in the determination of the $8-20 \mathrm{keV}$ luminosity function. Given this fact and taking into account the $L_{3-20} / L_{8-20}$ correction, the best-fit model obtained for the 8-20 keV luminosity function (Table 4) is consistent with the better constrained 3-20 keV luminosity function (Table 3). 
Table 4. 8-20 keV AGN luminosity function parameters.

\begin{tabular}{cccccccccc}
\hline \hline Sample & $\begin{array}{c}\log L_{3-20} \\
\mathrm{erg} \mathrm{s}^{-1}\end{array}$ & Size & $\begin{array}{c}\log L_{*}{ }^{a} \\
\mathrm{erg} \mathrm{s}^{-1}\end{array}$ & $\gamma_{1}$ & $\gamma_{2}$ & $\begin{array}{c}A \\
\mathrm{Mpc}^{-3}\end{array}$ & $\begin{array}{c}W_{8-20}(>41) \\
10^{38} \mathrm{erg} \mathrm{s}^{-1} \mathrm{Mpc}^{-3}\end{array}$ & $\begin{array}{c}N_{8-20}(>41) \\
10^{-4} \mathrm{Mpc}^{-3}\end{array}$ & $\begin{array}{c}P_{\mathrm{KS}} \\
\end{array}$ \\
\hline Total & $40.5-44.5$ & 37 & $42.82_{-0.50}^{+0.52}$ & $0.66_{-0.42}^{+0.32}$ & $1.98_{-0.28}^{+0.36}$ & $4.6(5.3) \times 10^{-6}$ & $3.6_{-0.6}^{+0.8}\left(4.3_{-0.7}^{+1.0}\right)$ & $10_{-5}^{+10}\left(12_{-6}^{+12}\right)$ & $>0.9$ \\
\hline
\end{tabular}

${ }^{a}$ All presented uncertainties are $1 \sigma$.

\section{AGN contribution to the local X-ray output}

In the previous section, we estimated the total energy released per unit volume in the 3-20 keV band by local AGNs with $L_{3-20}>10^{41} \mathrm{erg} \mathrm{s}^{-1}$ at $W_{3-20}(>41)=(5.2 \pm 1.2) \times$ $10^{38} \mathrm{erg} \mathrm{s}^{-1} \mathrm{Mpc}^{-3}$. The value and error given here take into account both the possible sample incompleteness and statistical uncertainty. Given the luminosity dependence of the absorption column density distribution discussed in Sect. 3, we can convert the above estimate to the standard $2-10 \mathrm{keV}$ band: $W_{2-10}(>40.7)=(2.9 \pm 0.7) \times 10^{38} \mathrm{erg} \mathrm{s}^{-1} \mathrm{Mpc}^{-3}$. Most of this emission is produced by Seyfert galaxies with X-ray luminosities below $\sim 10^{44} \mathrm{erg} \mathrm{s}^{-1}$.

It is interesting to compare the above value with the total $\mathrm{X}$-ray volume emissivity in the $2-10 \mathrm{keV}$ band, which has been estimated as $\rho_{2-10}=(6.5 \pm 1.9) \times 10^{38} \mathrm{erg} \mathrm{s}^{-1} \mathrm{Mpc}^{-3}$ (for $H_{0}=$ $75 \mathrm{~km} \mathrm{~s}^{-1} \mathrm{Mpc}^{-1}$ ) from the cross-correlation of IRAS galaxies with the HEAO-1 all-sky X-ray map (Miyaji et al. 1994). We may conclude from this comparison that sources other than classical Seyfert galaxies may provide similar contribution to the local X-ray emissivity. The obvious candidates are lowluminosity $\left(L_{2-10}<10^{41} \mathrm{erg} \mathrm{s}^{-1}\right)$ AGNs, starburst and nonactive galaxies and clusters of galaxies. In particular, the contribution of clusters of galaxies to the local $2-10 \mathrm{keV}$ emissivity is $\sim 0.5 \times 10^{38} \mathrm{erg} \mathrm{s}^{-1} \mathrm{Mpc}^{-3}$, as can be estimated from the measured 0.1-2.4 keV luminosity function (Böhringer et al. 2002) and the luminosity-temperature relation (Markevitch 1998).

We may further compare the local AGN volume emissivity with relevant estimates for a more distant Universe. The results of Cowie et al. (2003) obtained with the Chandra observatory indicate that the cumulative emission from AGNs with $L_{2-10}>$ $10^{42} \mathrm{erg} \mathrm{s}^{-1}$ (the actual energy range used was $2-8 \mathrm{keV}$ ) has decreased from a few $\times 10^{39} \mathrm{erg} \mathrm{s}^{-1} \mathrm{Mpc}^{-3}$ at $z=1-2$ to less than $10^{39} \mathrm{erg} \mathrm{s}^{-1} \mathrm{Mpc}^{-3}$ at $z \sim 0.5$. Our result thus suggests that a further decrease of the total energy production by AGNs has occured by the present epoch.

\section{Conclusions}

1. A well defined sample of 95 AGNs located at $|b|>10^{\circ}$, detected in the 3-20 keV band by the RXTE slew survey is presented. Most of the sources belong to the local population $(z<0.1)$. Accurate estimates of the intrinsic absorption column are presented for practically all of the sources.

2. The reconstructed $N_{\mathrm{H}}$ distribution of AGNs is drastically different for low-luminosity $\left(L_{3-20} \lesssim 10^{43.5} \mathrm{erg} \mathrm{s}^{-1}\right)$ and high-luminosity $\left(L_{3-20} \gtrsim 10^{43.5} \mathrm{erg} \mathrm{s}^{-1}\right)$ objects. Among the former, two thirds are X-ray absorbed $\left(10^{22} \mathrm{~cm}^{-2}<\right.$ $N_{\mathrm{H}}<10^{24} \mathrm{~cm}^{-2}$ ), whereas the corresponding fraction is less than $20 \%$ among the latter. These statistics do not take into account the population of Compton thick AGNs $\left(N_{\mathrm{H}}>\right.$ $10^{24} \mathrm{~cm}^{-2}$ ), to which our survey is not sufficiently sensitive.

3. The $3-20 \mathrm{keV}$ AGN luminosity function is derived, which is the best-to-date in quality at photon energies above $2 \mathrm{keV}$ for the local population. The luminosity function starts to flatten at $L_{3-20} \approx 10^{43.5} \mathrm{erg} \mathrm{s}^{-1}$ toward lower luminosities, approximately where obscured objects start to dominate the AGN population. A physical explanation for this behavior is required.

4. Comparison of the cumulative X-ray output of AGNs with $L_{3-20}>10^{41} \mathrm{erg} \mathrm{s}^{-1}$ with the previously estimated total X-ray volume emissivity in the local Universe demonstrates that low-luminosity $\left(L_{3-20}<10^{41} \mathrm{erg} \mathrm{s}^{-1}\right)$ AGNs, non-active galaxies and clusters of galaxies together may be emitting a similar amount of X-rays to Seyfert galaxies.

5. A sample of 35 unidentified sources - AGN candidates, detected during the RXTE slew survey is presented. 12 of these have a likely ROSAT soft X-ray counterpart with a better than 1 arcmin localization, so their identification in the optical and other bands should not be difficult. The positions of the other sources are currently known with a $1 \mathrm{deg}$ accuracy, and could be improved by scanning observations with X-ray telescopes.

Acknowledgements. We thank the anonymous referee for careful reading and constructive comments.

\section{References}

Awaki, H., Koyama, K., Inoue, H., \& Halpern, J. P. 1991, PASJ, 43, 195

Böhringer, H., Collins, C. A., Guzzo, L., et al. 2002, ApJ, 566, 93

Cowie, L. L., Barger, A. J., Bautz, M. W., Brandt, W. N., \& Garmire, G. P. 2003, ApJ, 584, L57

della Ceca, R., Palumbo, G. G. C., Persic, M., et al. 1990, ApJS, 72, 471

Ghosh, K. K., \& Soundararajaperumal, S. 1992, MNRAS, 259, 175

Grupe, D. 2000, New Astron. Rev., 44, 455

Guainazzi, M., Matt, G., Brandt, W. N., et al. 2000, A\&A, 356, 463

Ivezić, Z., Menou, K., Knapp, G. R., et al. 2002, AJ, 124, 2364

La Franca, F., Fiore, F., \& Vignali, C., et al. 2002, ApJ, 570, 100

Leighly, K. M. 1999, ApJS, 125, 317

Maiolino, R., \& Rieke, G. H. 1995, ApJ, 454, 95

Malizia, A., Bassani, L., Zhang, S. N., et al. 1999, ApJ, 519, 637

Markevitch, M. 1998, ApJ, 504, 27 
Matt, G., Pompilio, F., \& La Franca, F. 1999, New Astron., 4, 191

Matt, G., Fabian, A. C., Guainazzi, M., et al. 2000, MNRAS, 318, 173

Miyaji, T., Lahav, O., Jahoda, K., \& Boldt, E. 1994, ApJ, 434, 424

Piccinotti, G., Mushotzky, R. F., Boldt, E. A., et al. 1982, ApJ, 253, 485

Revnivtsev, M., Sazonov, S., Jahoda, K., \& Gilfanov, M. 2004, A\&A, 418, 927 (Paper I)

Reeves, J. N., \& Turner, M. J. L. 2000, MNRAS, 316, 234

Reynolds, C. S. 1997, MNRAS, 286, 513

Risaliti, G., Maiolino, R., \& Salvati, M. 1999, ApJ, 522, 157

Risaliti, G. 2002, A\&A, 386, 379

Schmid, H. M., Appenzeller, I., \& Burch, U. 2003, A\&A, 404, 505
Schmidt, M. 1968, ApJ, 151, 393

Schurch, N. J., \& Warwick, R. S. 2002, MNRAS, 334, 811

Steffen, A. T., Barger, A. J., Cowie, L. L., Mushotzky, R. F., \& Yang, Y. 2003, ApJ, 596, L23

Turner, T. J., \& Pounds, K. A. 1989, MNRAS, 240, 833

Tully, R. B. 1988, Nearby Galaxies Catalogue (Cambridge University Press)

Ueda, Y., Akiyama, M., Ohta, K., \& Miyaji, T. 2003, ApJ, 598, 886 Wolter, A., Comastri, A., Ghisellini, G., et al. 1998, A\&A, 335, 899

Yaqoob, T. 1997, ApJ, 479, 184

Young, A. J., \& Wilson, A. S. 2004, ApJ, 601, 133 